\title{
Performance Investigation of Modified Desert Cooler
}

\author{
Dr.J P Yadav ${ }^{1}$, Pankaj Sharma ${ }^{2}$ \\ ${ }^{1}$ Professor: B S Dr B R A College of Agricultural Engineering \& Technology, Etawah - 206001 (UP), India \\ ${ }^{2}$ Assistant Professor: B S Dr B R A College of Agricultural Engineering \& Technology, Etawah - 206001 (UP), \\ India
}

ABSTRACT: Desert cooler is effective and efficient when the dry bulb temperature is high and the relative humidity is low, restricting its use to dry climates. Traditionally, desert cooler pads consist of wood wool, coconut coir or khus inside containment net. Now-a-day cel-dek pads are also very much in use because of its high efficiency of cooling.In desert cooler as the relative humidity increases with more moisture content in the air, the comfort level goes down even though the dry bulb temperature is not so high. In this paper to overcome this, the heating-coil is used tocontrol the humidity level of the room by increasing the temperature of the air by placing a heating coil across the cooling pad for summer cooling. In the winter season the same heating-coil can be used as a blower for increasing temperature of room. The experimental results with modified desert cooler reveals that temperature level decreases up to $7^{\circ} \mathrm{C}$ and humidity of the room increase by $20 \%$ but here the humidity is decreased with slightincrease in temperature to obtain the approximately required comfort zone which is suitable for the human being.

Keywords: Cooling pads, Cooling efficiency, desert cooler, heating-coil etc.

\section{INTRODUCTION}

A desert cooler is a device that cools air through the evaporation of water. Evaporative cooling differs from typical air conditioning systems which use vapour compression or absorption refrigeration cycles. Evaporative cooling works by employing water's large enthalpy of vaporization. The temperature of dry air can be dropped significantly through the phase transition of liquid water to water vapour (evaporation), which can cool the air using much less energy than refrigeration.In extremely dry climates, evaporative cooling of air has the added benefit of conditioning the air with more moisture for the comfort of building occupants. The cooling potential for evaporative cooling is dependent on the wet bulb depressionthat is the difference between dry bulb temperature and wet bulb temperature.

Desert cooler involves converting sensible heat to latent heat by evaporating water to remove heat from the air. Energy for evaporation of water is provided by the sensible heat in air. Desert coolers lower the temperature of air using the process of the cooling and Dehumidification. Desert cooler is the addition of water vapour into air, which causes lowering the temperature of the air. The energy needed to evaporate the water is taken from the air in the form of the sensible heat, which affects the temperature of the air, and converted into the latent heat. This conversion of sensible heat to the latent heat is known as adiabatic process because it occurs at a constant enthalpy value.Evaporative cooling technique is very old and easy method of cooling. Humans have been using this technique for their comfort and convenience. In many old monuments we can find this type of cooling technique but because of systems inefficiency and some constraints we are not using this technique so in this paper focus is made to understand the system working and improve its efficiency by using latest cooling pad and also make a study how to design desert cooler for a particular region. Some of the researchers have worked on the desert cooler whose findings are reported in the ensuing paragraphs.

Faleh Al-Sulaiman (2002) says that Desert cooler can use various fibres like date palm fibres, jute and wood pad for wetted cooler pads and evaluated the performance resulted just has maximum cooling efficiency.Kothare and Borker (2011) studied and reported that a "Modified Desert Cooler" which cools the air more efficiently than the conventional desert cooler as well as provide coldpure water for drinking purpose. It also decreased moisture content of the air coming through desert cooler up to some extent.Khond (2011) used pads of stainless steel wiremesh; coconut-coir, khus and wood wool were fabricated and tested using a laboratory-scale experimental arrangement. Minimum water consumption was observed in stainless steel wire mesh pad $(0.066 \mathrm{Lit} / \mathrm{min})$ at same fan speed. Stainless steel wire mesh pad $(4.5 \mathrm{~m} / \mathrm{s})$ and coconut coir pad $(5.2 \mathrm{~m} / \mathrm{s})$ had shown higher air velocity which provides proper air distribution in room while wood wool pad $(4 \mathrm{~m} / \mathrm{s})$ and khus pad (3.4 $\mathrm{m} / \mathrm{s}$ ) were shown lower velocity. Maximum and minimum cooling efficiency were found in wood wool pad and stainless steel wire mesh pad.Poonia MP,BharadwajA, developed a cooler cum refrigerator which can be utilized for the purpose of 
air cooling, drinking water cooling viz storing the vegetables and medicines without altering the performance of desert cooler. The energy saving by doing so is saved more than $30 \mathrm{~W}$. A small size desert cooler can cool more than 24 litre water per day up to the wet bulb temperature of outside air as well as could store vegetables for more than five days.Vivek W Khond mentions evaporative cooling requires an abundant water source as it evaporate, and is only efficient when the relative humidity is low, restricting its effective use to dry climates.

\section{RakeshRoshan\&UpendraParashar}

(2015)workedwith a cotton based cooling pad as evaporator. An extra fan has been used to have effective evaporation. In his investigation four differenttemperatureswere taken. During the experiment an excellent result was noticed. Almost same bypass factor was observed forth half the value of water consumption by the existing desert cooler.

MShariaty-Niassar, N. Gilaniinvestigated different types of indirect evaporativecoolers(IECs)using the CFD technique. Several codes were defined in MATLABfor modelling the parallel flow, counter flow and cross flow layout. The calculated results showthat when the air relative humidity is lower than $70 \%$, the system can prepare a goodindoor condition even at $50^{\circ} \mathrm{C}$, and a higher performance is achieved by using the IECwith counter current configuration. The results showed that IECs can be successfullyused in hot and humid climates to fulfil the indoor thermal comfort conditions.

\section{WORKING PRINCIPLE}

The principle on which a desert cooler works is evaporative cooling. Evaporative cooling is a process in which sensible heat is removed and moisture added to air. When air passes through a spray of water it gives up heat to water, some of water evaporates and picks up heat from the air equivalent to its latent heat. Thus the vapour formedis carried along in air stream. In the way air is cooled and dehumidified. Desert cooler is suitable for place where the humidity is quite low and temperature quite high. The conditions are in conformity with desert areas. Hence the coolers are called desert coolers. The water is filled in the sump of the cooler. A water pump lifts the water and supplies it at the top of the cooler to the water distribution system which consist of small branches water pipe which deliver equal amount of water to the troughs which in turns supply water to the wetted pad. The water which drops back from the pads is re-circulated. The blower pulls the air through the wetted pads and deliversit to space to be cooled through an opening in the fourth side of the cabinet of desert cooler. The air which is sucked through the pads is cooled by the principle of evaporative cooling. The fan/blower gives adequate velocity to the air before it is delivered to the spaces to be cooled.To have long life of the desert cooler and better performance, pads should be changed every year and holes for water distribution system should be cleaned. Some of the important formula used to analyze the performance of desert cooler is given as below:

$\mathrm{W}=0.622 \times \frac{p_{v}}{p_{b-p_{v}}}$

Where $p_{v}=$ partial pressure of water vapour, $p_{b}=$ Barometric Pressure, $\mathrm{W}=\mathrm{sp}$. humidity or humidity ratio

$\emptyset=\frac{\mathrm{m}_{\mathrm{v}}}{\mathrm{m}_{\mathrm{s}}}=\frac{\mathrm{p}_{\mathrm{v}}}{\mathrm{p}_{\mathrm{s}}}$

Where $\emptyset=$ relative humidity, $\mathrm{m}_{\mathrm{v}}=$ Actual Mass of Water Vapour, $m_{s}=$ Volume of Moist air to the mass of water vapour

$\rho_{v}=\frac{W\left(p_{b-} p_{v}\right)}{R_{a} \times T_{d}}$

Where $\rho_{\mathrm{v}}=$ vapour density or absolutely humidity, $\mathrm{R}_{\mathrm{a}}=$ Gas Constant for Air $=0.287 \mathrm{~kJ} / \mathrm{kg} \mathrm{K}$, $\mathrm{T}_{\mathrm{d}}=$ Dry Bulb Temperature in $\mathrm{K}$

$\mu=\frac{\mathrm{w}}{\mathrm{w}_{\mathrm{s}}}=\frac{\mathrm{p}_{\mathrm{v}}}{\mathrm{p}_{\mathrm{s}}} \frac{\left(\mathrm{p}_{\left.\mathrm{b}-\mathrm{p}_{\mathrm{s}}\right)}\right.}{\left(\mathrm{p}_{\left.\mathrm{b}-\mathrm{p}_{\mathrm{v}}\right)}\right)}$

Where $W_{s}=$ Specific Humidity of Saturated air, $\mu=$ percentage humidity

$m_{a}=\frac{p_{a} v}{R_{a} T_{d}}$

Where $m_{a}=$ mass of dry air

$m_{v}=\mathrm{W} \times m_{a}$

Where $m_{v}=$ mass of water vapour

$\mathrm{h}=1.022 t_{d}+\mathrm{W}\left(\mathrm{h}_{\mathrm{fgdp}}+2.3 \mathrm{t}_{\mathrm{dp}}\right)$

Where $\mathrm{h}=$ enthalpy

\section{DESCRIPTION OF EXPERIMENTAL SET UP}

The experimental set up is shown at figure 3.1. The different parts used in set up are as electric motor, fan, water pump, sump,heating-coil,switch andcooling pads,mostly the wood wool \&coconut coir are used because this gives more cooling efficiency than steel wire mesh, khus pad or synthetic foam sheet but now-a-days there are new pads available called as cel-dek pads which gives very high efficiency of cooling near about 90\%.Two bulbsare used for controlling the speed of fan so that revolution of fan is at minimum. When one bulb is switched'on' the speed of fan is lowered compare to normal condition and when both bulbsare switched'on' the speed of fan is very low. Bulbsare also used to increase temperature of the air.The air sucked by the fan from all three sides and gets cooled passing through the pads. Cooled and dehumidified air passes through the heating-coil where it maintains the dry bulb temperature and relative humidity of air.Heatingcoil -- sensible or simple heating of air takes place when it flows over a heating-coil similar to the cooling and dehumidifying coil whose surface 
temperature is higher than dry bulb temperature of air. Heating-coil is done to bring the air to the designed dry bulb temperature and relative humidity.

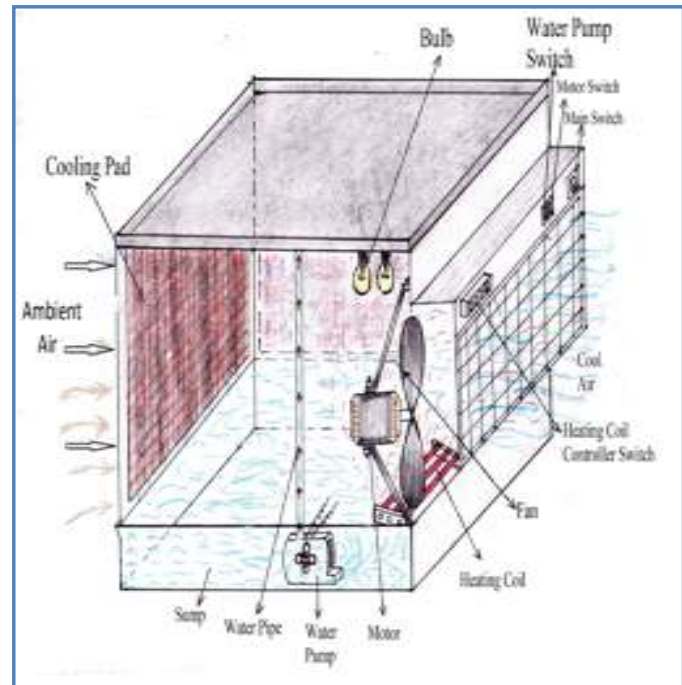

Figure 3.1:Schematic Diagram of Desert Cooler

\section{HUMAN BODY PARAMETERS}

In the case of human being the heat stored in the body has maximum and minimum limits which when exceeded brings death. The usual body temperature, for a normal man (whenthe heat stored, $\mathrm{Q}_{\mathrm{s}}=0$ ) is $37^{\mathrm{O}} \mathrm{C}$. The temperature of the body when falls below $36.5^{\circ} \mathrm{C}$ and exceeds $40.5^{\circ} \mathrm{C}$ is dangerous. There is some kind of thermostatic control called vasomotor control mechanism in the human body which maintains the temperature of body at the normal level of $37^{\circ} \mathrm{C}$, by regulating the blood supply to the skin. When the temperature of the body falls (i.e. the heat stored $\mathrm{Q}_{\mathrm{s}}$ in the body is negative), then the vasomotor control decreases the circulation of blood which decreases conductivity of of nerve cells and other tissues between the skin and the inner body cell. This allows skin temperature to fall but allows higher inner temperature of body cells beneath. When the temperature of the body rises (i.e. the heat stored $\mathrm{Q}_{\mathrm{s}}$ in the body is positive), then the vasomotor control increases blood circulation which increases conductivity of tissues and hence allows less temperature drop between the skin and inner body cells. The human body feels comfortable when there is no change in the body temperature, i.e. when the heat stored in the body $\mathrm{Q}_{\mathrm{s}}$ is zero. Any variation in the body temperature acts as a stress to the brain which ultimately results in either perspiration or shivering.

\subsection{Heat and Moisture Losses from the Human \\ Body}

The heat given off from human body as either sensible or latent heat or both. In order to design any air-conditioning system for spaces which human bodies are to occupy, it is necessary to know at which these two forms of heat are given off under different conditions of air temperature and bodily activity. Figure (a) below shows the graph between sensible heat loss by radiation and convection for an average man and the dry bulb temperature for different types of activity. Figure (b) shows the graphbetween the latent heat loss by evaporation for an average man and dry bulb temeperature for different type of activity.

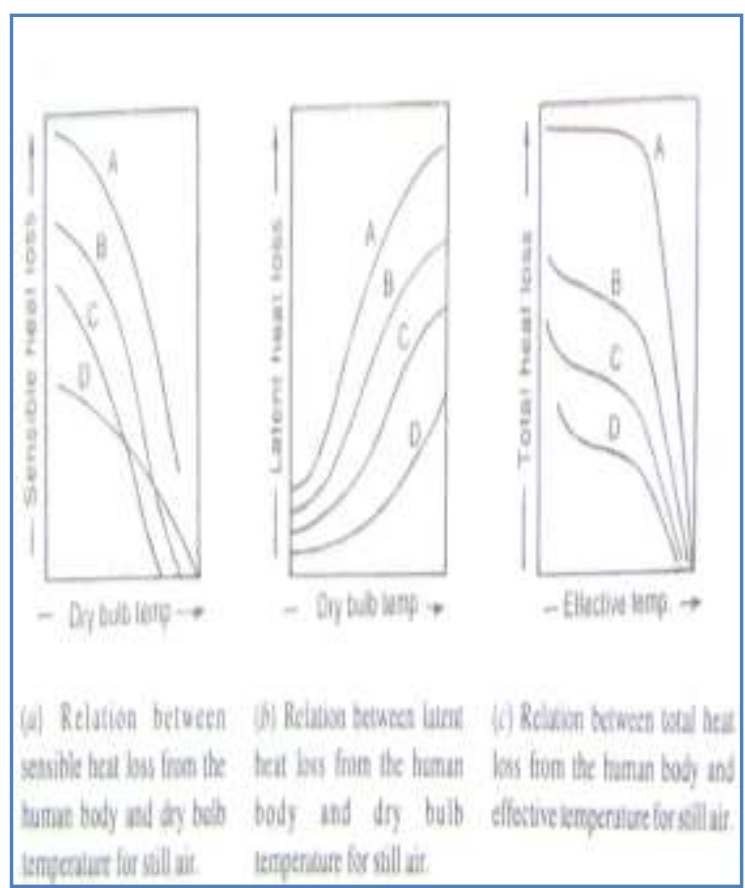

Figure 4.1: Variation between heat loss and DBT/ Effective temperature

The total heat loss from the human body under varying effective temperatures is shown in figure (c). From curve D, which applies to men at rest, we see that from about $19^{\circ} \mathrm{C}$ to $30^{\circ} \mathrm{C}$ effective temperature, the heat loss is constant. At the lower effective temperature, the heat dissipation increases which results in a feeling of coolness. At higher effective temperature, the ability to lose the heat rapidly decreases resulting in severe discomfort.

The curves $A, B, C$ and $D$ shown in figure represents as follows:

Curve A - Men working at the rate of $90 \mathrm{kN}-\mathrm{m} / \mathrm{h}$ Curve B - Men working at the rate of $45 \mathrm{kN}-\mathrm{m} / \mathrm{h}$ Curve C - Men working at the rate of $22.5 \mathrm{kN}-\mathrm{m} / \mathrm{h}$ Curve D - Men at rest 


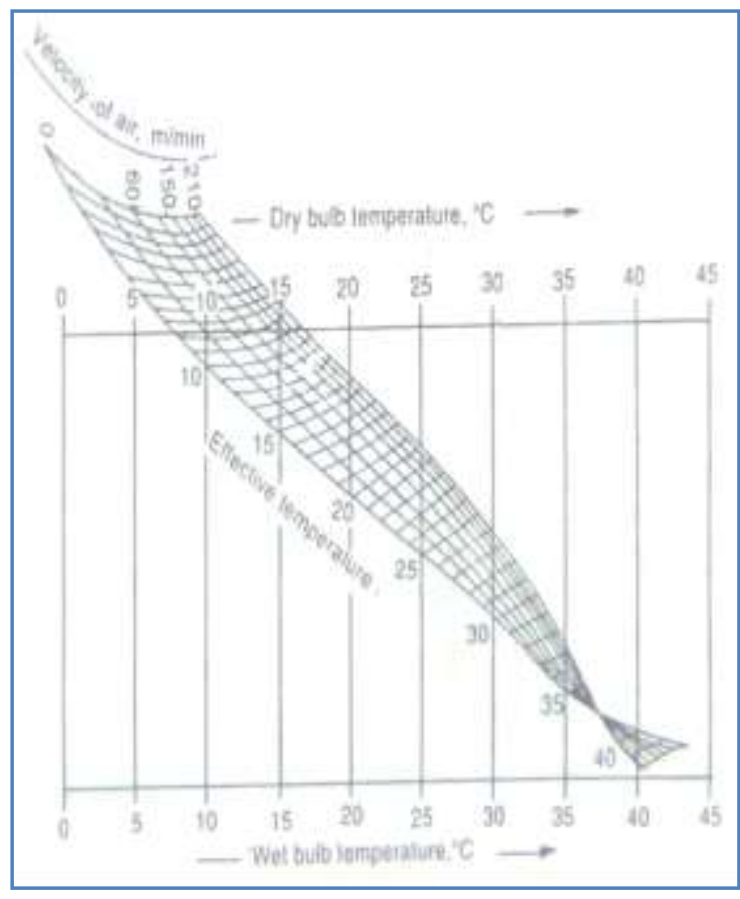

Figure4.2: Variation of effective temperature with air velocity

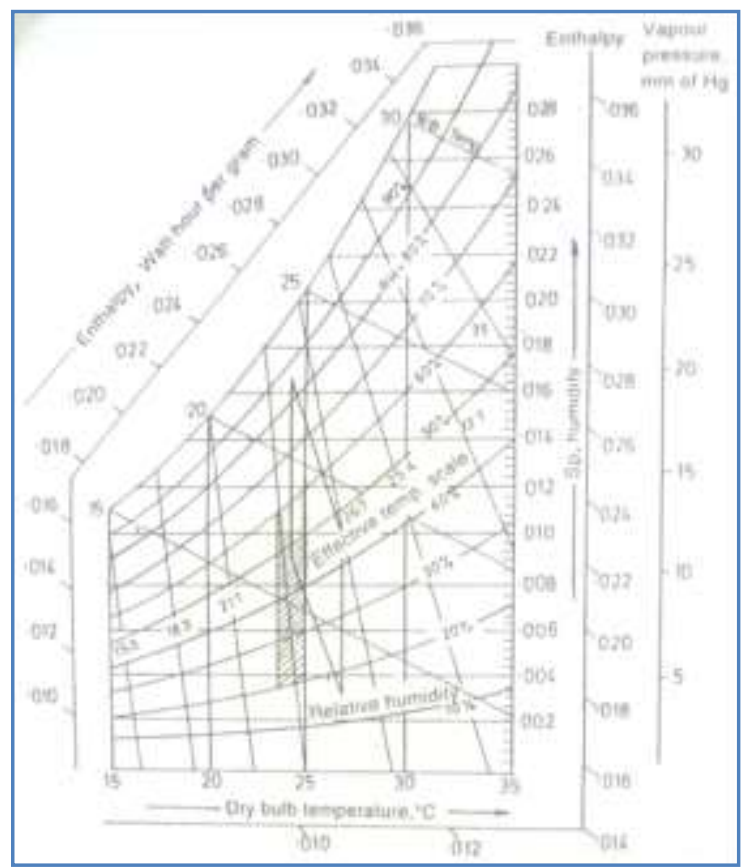

Figure 4.3:Modified comfort chart

\section{ANALYSIS OF PSYCHROMETRIC PROPERTIES}

During analysis at room temperature the Dry Bulb Temperature is as high as $30-40^{\circ} \mathrm{Cwith}$ relative humidity as less as $30-50 \%$. When switch 'on' the cooler thedry bulb temperature is less than that at room temperature with comparatively increased relative humidity $(70-80 \%)$ but when one heating- coil with cooling pad is put 'on' DBT is slightly increased. In the respect of DBT, relative humidity is also slightly decreased to obtain required comfort zone.

The observations of moist air coming out from desert cooler in the month of April and May 2016 are at tabled 5.1-5.8 above. The values of dry bulb temperature, relative humidity, wet bulb temperature, specific humidity, dew point temperature, enthalpy, vapour pressure and specific volume measured/ calculated at room temperature between 9:00 am to 6:00 pm applying cooling/ heating coil separately or jointly. The italic and high-lighted data shown at tables 1-8 below comes under comfortable zone as for human comfort the DBT and RH should be between $22-25^{\circ} \mathrm{C}$ and $20-60 \%$.

Table No:5.1 (05 April 2016)

\begin{tabular}{|c|c|c|c|c|c|c|c|c|}
\hline 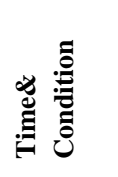 & 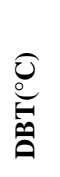 & 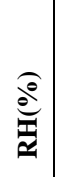 & 它 & $\underbrace{30}_{3}$ & 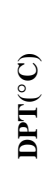 & 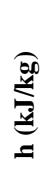 & 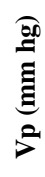 & 总 \\
\hline 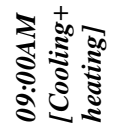 & $\begin{array}{l}\text { ?n} \\
\stackrel{?}{i}\end{array}$ & हैं & $\stackrel{\widehat{\infty}}{\stackrel{i}{\sim}}$ & $\stackrel{\stackrel{\hat{S}}{\mathrm{i}}}{ }$ & 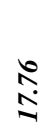 & కั๋ & $\frac{\pi}{5}$ & 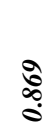 \\
\hline 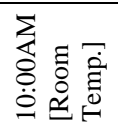 & $\begin{array}{l}\text { त్ } \\
\text { ర్లు }\end{array}$ & $\stackrel{\tilde{q}}{\tilde{q}}$ & $\begin{array}{l}\dot{0} \\
\dot{i}\end{array}$ & $\overrightarrow{\mathrm{I}}$ & 甬 & $\begin{array}{l}\text { हे } \\
\text { in }\end{array}$ & 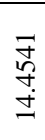 & $\underset{\substack{\vec{\alpha} \\
0}}{\overrightarrow{0}}$ \\
\hline 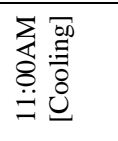 & $\stackrel{P}{\stackrel{P}{+}}$ & $\begin{array}{l}0 \\
\dot{0} \\
0\end{array}$ & ले & $\stackrel{m}{\rightarrow}$ & 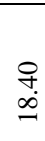 & 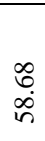 & $\begin{array}{l}\hat{R} \\
\infty \\
\\
\underline{n}\end{array}$ & 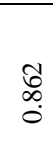 \\
\hline 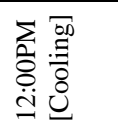 & $\begin{array}{l}\text { ñ } \\
\text { ñ }\end{array}$ & $\stackrel{i}{r}$ & 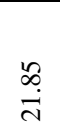 & $\begin{array}{l}\text { of } \\
\text { in }\end{array}$ & 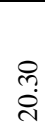 & $\begin{array}{l}\text { के } \\
\vec{b}\end{array}$ & $\begin{array}{l}\qquad \\
\infty \\
\stackrel{\Xi}{\simeq}\end{array}$ & $\begin{array}{l}8 \\
0 \\
0 \\
0\end{array}$ \\
\hline
\end{tabular}

Table No:5.2 (12 April 2016)

\begin{tabular}{|c|c|c|c|c|c|c|c|c|}
\hline 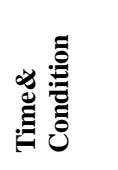 & 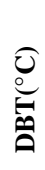 & $\stackrel{0}{\approx}$ & है: & $\underbrace{+\infty}_{3}$ & 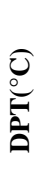 & = & 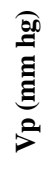 & हैं \\
\hline 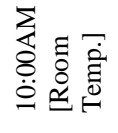 & $\overline{\dot{m}}$ & $\begin{array}{l}n \\
\infty \\
\infty\end{array}$ & $\stackrel{\infty}{\stackrel{i}{े}}$ & $\stackrel{\sigma}{\varrho}$ & $\begin{array}{l}\tilde{n} \\
\text { ñ. }\end{array}$ & $\begin{array}{l}\overrightarrow{7} \\
\text { in }\end{array}$ & 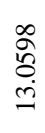 & $\infty$ \\
\hline 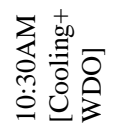 & $\begin{array}{l}\dot{0} \\
\dot{\sim}\end{array}$ & $\stackrel{\sqrt[3]{2}}{\stackrel{2}{2}}$ & $\begin{array}{l}\infty \\
\stackrel{\infty}{+} \\
\stackrel{+}{*}\end{array}$ & $\begin{array}{l}\overrightarrow{0} \\
\infty \\
-\infty\end{array}$ & $\begin{array}{l}\stackrel{8}{\mathrm{~d}} \\
\vec{i}\end{array}$ & $\begin{array}{l}\tilde{n} \\
n \\
n\end{array}$ & $\begin{array}{l}\stackrel{\infty}{\circ} \\
\stackrel{\vec{\lambda}}{\Delta}\end{array}$ & $\infty$ \\
\hline 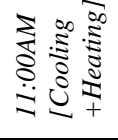 & iे & 8 & Ș & 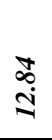 & $\stackrel{\infty}{\circledR}$ & $\begin{array}{l}2 \\
\infty \\
\infty\end{array}$ & $\begin{array}{l}\bar{m} \\
\vdots \\
=\end{array}$ & \\
\hline
\end{tabular}


Table No:5.3 (19 April 2016)

\begin{tabular}{|c|c|c|c|c|c|c|c|c|}
\hline 岂 & 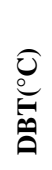 & 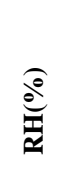 & 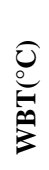 & $\underbrace{\stackrel{000}{50}}_{3}$ & 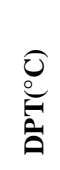 & 尊 & 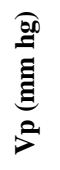 & 总 \\
\hline 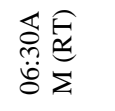 & 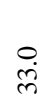 & ñ & 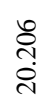 & $\vec{b}$ & 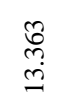 & $\underset{i}{\stackrel{i}{i}}$ & $\stackrel{\vec{n}}{=}$ & $\begin{array}{l}\mathscr{D} \\
\infty \\
\infty \\
0\end{array}$ \\
\hline 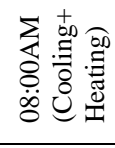 & mi & $\begin{array}{l}n \\
0 \\
0\end{array}$ & $\begin{array}{l}\text { nे } \\
\text { ते }\end{array}$ & $\stackrel{\vec{\sigma}}{\hat{0}}$ & 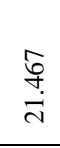 & $\begin{array}{l}8 \\
8 \\
8\end{array}$ & $\begin{array}{l}\stackrel{0}{3} \\
\stackrel{\pi}{2}\end{array}$ & $\begin{array}{l}0 \\
\infty \\
0 \\
0\end{array}$ \\
\hline 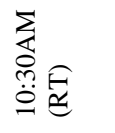 & $\stackrel{\stackrel{H}{m}}{m}$ & ले & 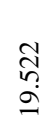 & 年 & 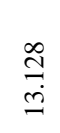 & $\begin{array}{l}\text { o } \\
\text { v } \\
i n\end{array}$ & 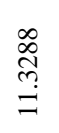 & $\begin{array}{l}n \\
\infty \\
\infty \\
0 \\
0\end{array}$ \\
\hline 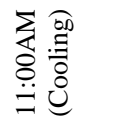 & $\underset{\stackrel{d}{\sim}}{ }$ & : & $\begin{array}{l}\overrightarrow{\vec{b}} \\
\vec{i}\end{array}$ & 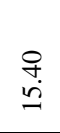 & 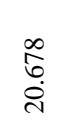 & \begin{tabular}{l}
\multirow{2}{0}{} \\
0 \\
0 \\
0
\end{tabular} & $\begin{array}{l}\widehat{\widehat{A}} \\
\stackrel{\infty}{-}\end{array}$ & $\begin{array}{l}\mathcal{M} \\
\infty \\
0 \\
0\end{array}$ \\
\hline 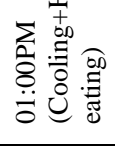 & ๙ & $\stackrel{?}{i}$ & $\underset{\mathbb{i}}{\mathbf{d}}$ & $\underset{\ddagger}{\ddagger}$ & ڤ్ & $\begin{array}{l}\stackrel{0}{0} \\
\hat{j}\end{array}$ & 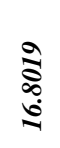 & $\stackrel{2}{2}$ \\
\hline
\end{tabular}

Table No:5.4 (28 April 2016)

\begin{tabular}{|c|c|c|c|c|c|c|c|c|}
\hline 泀 & 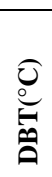 & $\stackrel{\widehat{d}}{\widehat{d}}$ & 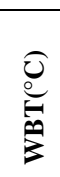 & $\frac{\sqrt{80}}{30}$ & 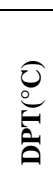 & : & 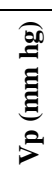 & 尊 \\
\hline $\begin{array}{l}\sum_{0} \\
\stackrel{0}{0} \widehat{E} \\
\stackrel{0}{0}\end{array}$ & $\stackrel{m}{\stackrel{0}{d}}$ & in & $\frac{\mathfrak{N}}{\stackrel{N}{N}}$ & $\stackrel{\mathscr{a}}{\mathfrak{s}}$ & $\stackrel{0}{\stackrel{0}{\circ}}$ & $\frac{\sqrt[5]{6}}{6}$ & 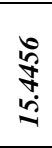 & 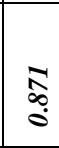 \\
\hline 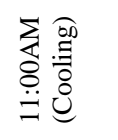 & $\begin{array}{l}n \\
\text { ñ }\end{array}$ & $\stackrel{\leftrightarrow}{i}$ & $\begin{array}{l}\tilde{N} \\
\stackrel{\vec{\lambda}}{\alpha}\end{array}$ & $\begin{array}{l}n \\
\stackrel{n}{a} \\
n\end{array}$ & 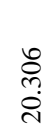 & $\begin{array}{l}\text { ठे } \\
\text { ठే }\end{array}$ & 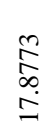 & $\begin{array}{l}0 \\
0 \\
0 \\
0 \\
0\end{array}$ \\
\hline 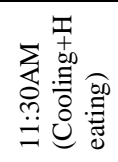 & $\stackrel{\dot{m}}{\circ}$ & กี่ & $\begin{array}{l}\text { t: } \\
\text { dे }\end{array}$ & $\begin{array}{l}\vec{\alpha} \\
\vdots \\
\vdots\end{array}$ & $\stackrel{\tilde{i}}{\mathbf{i}}$ & $\begin{array}{l}\stackrel{\Omega}{R} \\
\stackrel{R}{R}\end{array}$ & $\begin{array}{l}\hat{5} \\
0 \\
0 \\
\stackrel{0}{0}\end{array}$ & $\underset{\infty}{\infty}$ \\
\hline 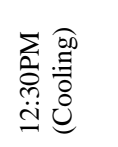 & ปे & $\begin{array}{l}n \\
\text { : } \\
\text { f }\end{array}$ & $\begin{array}{l}\stackrel{\infty}{+} \\
\stackrel{i}{\sim}\end{array}$ & $\begin{array}{l}\infty \\
\stackrel{0}{\Xi} \\
\end{array}$ & 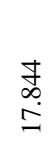 & $\begin{array}{l}\text { o. } \\
\text { o. } \\
\text { in }\end{array}$ & $\begin{array}{l}\widetilde{\pi} \\
\stackrel{n}{n} \\
\stackrel{2}{2}\end{array}$ & $\begin{array}{c}\widetilde{1} \\
\infty \\
0 \\
0\end{array}$ \\
\hline
\end{tabular}

Table No:5.5 (03 May 2016)

\begin{tabular}{|c|c|c|c|c|c|c|c|c|}
\hline 葛 & 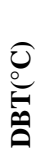 & 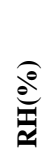 & है & है & 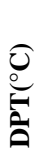 & : & 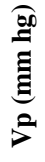 & 造 \\
\hline 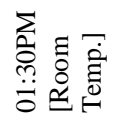 & $\tilde{j}$ & $\begin{array}{l}n \\
\stackrel{n}{n} \\
n\end{array}$ & $\begin{array}{l}\text { ñ } \\
\text { ñ. }\end{array}$ & 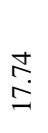 & $\begin{array}{l}\text { ડેे } \\
\text { הે }\end{array}$ & $\begin{array}{c}\underset{N}{\infty} \\
\stackrel{\infty}{r}\end{array}$ & $\begin{array}{l}\overrightarrow{0} \\
\hat{ᄋ} \\
\text { ेे }\end{array}$ & $\mid \begin{array}{l}\bar{\alpha} \\
\dot{0}\end{array}$ \\
\hline 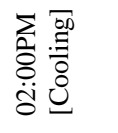 & ¿̊. & 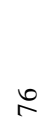 & $\begin{array}{l}\text { aे. } \\
\text { i. }\end{array}$ & ثे. & $\begin{array}{l}\hat{a} \\
\dot{d}\end{array}$ & $\begin{array}{l}\hat{\alpha} \\
\stackrel{\infty}{\infty}\end{array}$ & $\begin{array}{l}\text { గ్ } \\
\text { तెं }\end{array}$ & $\mid \begin{array}{l}\infty \\
\infty \\
0 \\
0\end{array}$ \\
\hline 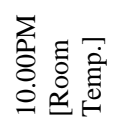 & $\stackrel{m}{m}$ & ले & $\begin{array}{l}\tilde{n} \\
\text { הิ }\end{array}$ & $\begin{array}{l}\stackrel{P}{\mathrm{I}} \\
\text { (n) }\end{array}$ & 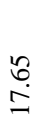 & $\begin{array}{l}\frac{n}{0} \\
\text { bi }\end{array}$ & $\begin{array}{l}\vec{n} \\
\vec{n}\end{array}$ & $\mid \begin{array}{c}0 \\
\infty \\
\infty \\
0\end{array}$ \\
\hline 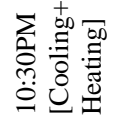 & $\stackrel{\mathrm{i}}{\mathrm{s}}$ & oे & $\begin{array}{l}\bar{\sigma} \\
\stackrel{i}{\vec{j}}\end{array}$ & 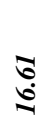 & $\stackrel{\widehat{\phi}}{\stackrel{\sim}{\sim}}$ & 命 & 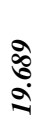 & 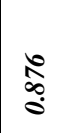 \\
\hline 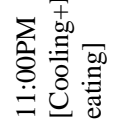 & $\hat{\imath}$ & 80 & त̂ & 10 & 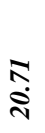 & ه్ & m & $\stackrel{\mathbb{\alpha}}{\stackrel{\infty}{S}}$ \\
\hline
\end{tabular}

Table No: 5.6 (10 May 2016)

\begin{tabular}{|c|c|c|c|c|c|c|c|c|}
\hline 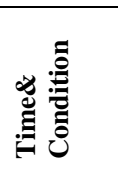 & 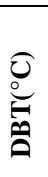 & $\frac{\widehat{e}}{\pi}$ & 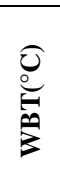 & $\frac{100}{30}$ & 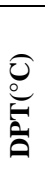 & 送 & & 总 \\
\hline 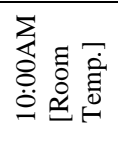 & $\frac{1}{m}$ & 8 & 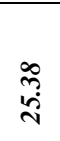 & $\stackrel{\infty}{\infty}$ & ָे & $\stackrel{\hat{尺}}{\hat{R}}$ & बें & $\mid \begin{array}{c}\infty \\
\infty \\
\infty\end{array}$ \\
\hline 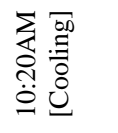 & 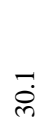 & $R$ & $\begin{array}{l}\hat{n} \\
\text { ते }\end{array}$ & $\begin{array}{l}\stackrel{\partial}{\partial} \\
\infty \\
\infty\end{array}$ & $\begin{array}{l}\text { Oे } \\
\dot{d}\end{array}$ & $\stackrel{\substack{m \\
\infty}}{\infty}$ & $\stackrel{f}{\stackrel{f}{d}}$ & $\mid \begin{array}{c}\infty \\
\infty \\
\infty \\
0\end{array}$ \\
\hline 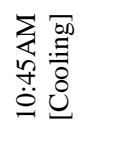 & 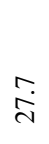 & $\begin{array}{l}n \\
\infty \\
\infty\end{array}$ & $\begin{array}{l}\stackrel{a}{+} \\
\dot{d}\end{array}$ & $\stackrel{\vec{\Xi}}{\stackrel{\square}{\circ}}$ & $\begin{array}{l}\stackrel{2}{+} \\
\dot{d}\end{array}$ & 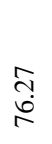 & $\frac{d}{\stackrel{d}{N}}$ & $\mid \begin{array}{c}\infty \\
\infty \\
\infty \\
0\end{array}$ \\
\hline 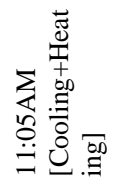 & in & $\stackrel{2}{2}$ & 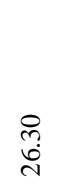 & तิ & 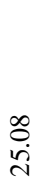 & $\stackrel{\infty}{\infty}$ & $\begin{array}{l}\infty \\
\text { తi } \\
\text { in }\end{array}$ & $\begin{array}{l}\infty \\
\infty \\
\infty\end{array}$ \\
\hline
\end{tabular}


Table No:5.7 (15 May 2016)

\begin{tabular}{|c|c|c|c|c|c|c|c|c|}
\hline 总 & 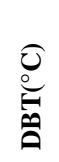 & $\stackrel{\overbrace{}}{\stackrel{0}{\approx}}$ & 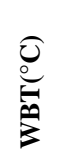 & 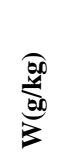 & 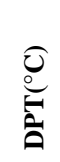 & 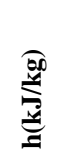 & 绨 & 总 \\
\hline 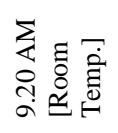 & $\begin{array}{l}n \\
n \\
n\end{array}$ & $\hat{\circ}$ & $\hat{\phi}$ & $\begin{array}{l}\tilde{B} \\
\infty \\
\infty\end{array}$ & $\underset{i}{\vec{i}}$ & $\underset{\infty}{\stackrel{J}{\sigma}}$ & $\begin{array}{l}\text { fे } \\
\text { त्र }\end{array}$ & \&̊. \\
\hline 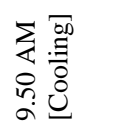 & nे & $\stackrel{n}{\stackrel{n}{r}}$ & $\begin{array}{l}\text { 克 } \\
\text { d. }\end{array}$ & 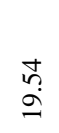 & $\begin{array}{l}\stackrel{\leftrightarrow}{+} \\
\stackrel{+}{+}\end{array}$ & $\begin{array}{l}\frac{0}{n} \\
\stackrel{2}{2}\end{array}$ & $\begin{array}{l}\tilde{0} \\
0 \\
0 \\
\tilde{i}\end{array}$ & $\begin{array}{l} \pm \\
\infty \\
\infty \\
0\end{array}$ \\
\hline 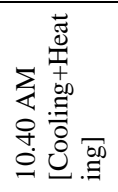 & & $\begin{array}{l}n \\
\text { nु }\end{array}$ & $\begin{array}{l}\stackrel{n}{\sim} \\
\underset{\sim}{\sim}\end{array}$ & $\stackrel{R}{\stackrel{R}{6}}$ & $\begin{array}{l}\vec{\triangleright} \\
\stackrel{\vec{\nu}}{2}\end{array}$ & $\begin{array}{l}\mathbb{N} \\
\stackrel{i}{i}\end{array}$ & 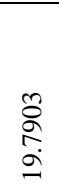 & $\begin{array}{c}\vec{\infty} \\
\infty \\
0\end{array}$ \\
\hline 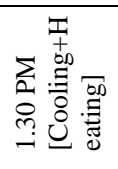 & ?. & के & $\begin{array}{l}\bar{\alpha} \\
\tilde{\vec{j}}\end{array}$ & $\stackrel{7}{s}$ & 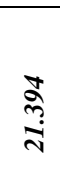 & 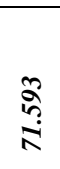 & 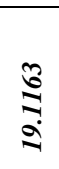 & $\underset{\infty}{\infty}$ \\
\hline 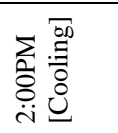 & $\widehat{\stackrel{d}{d}}$ & 13 & $\begin{array}{l}\text { 心ิ } \\
\text { ָे }\end{array}$ & బै & ते & $\stackrel{\infty}{:}$ & $\frac{\sqrt{5}}{2}$ & 今̂ \\
\hline
\end{tabular}

Table No:5.8 (17 May 2016)

\begin{tabular}{|c|c|c|c|c|c|c|c|c|}
\hline 竞 & 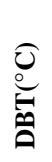 & $\stackrel{0}{\stackrel{0}{*}}$ & 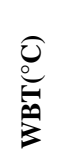 & 焉 & $\frac{\partial}{\mathscr{O}}$ & 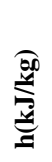 & 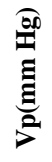 & 递 \\
\hline 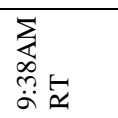 & 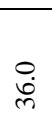 & $\stackrel{\sim}{m}$ & $\begin{array}{l}\tilde{n} \\
\stackrel{\lambda}{n} \\
\end{array}$ & $\stackrel{n}{=}$ & $\begin{array}{l}\tilde{N} \\
\text { } \\
\text { }\end{array}$ & 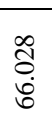 & $\begin{array}{l}\text { ה్ } \\
\text { aे } \\
\stackrel{-}{2}\end{array}$ & $\begin{array}{c}\tilde{\sigma} \\
\stackrel{\alpha}{0} \\
0\end{array}$ \\
\hline 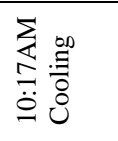 & 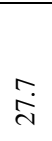 & $\stackrel{\circ}{\vec{r}}$ & $\begin{array}{l}n \\
\omega \\
\tilde{n} \\
\tilde{i}\end{array}$ & $\begin{array}{l}R \\
\stackrel{0}{6}\end{array}$ & $\begin{array}{l}\vec{\delta} \\
\stackrel{\vec{\lambda}}{\alpha}\end{array}$ & $\stackrel{\text { }}{\stackrel{\rho}{R}}$ & $\begin{array}{l}\stackrel{2}{2} \\
\stackrel{2}{2}\end{array}$ & $\begin{array}{c}n \\
\substack{n \\
\infty \\
0}\end{array}$ \\
\hline 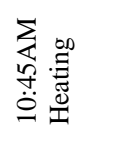 & ஓें & in & $\begin{array}{l}\stackrel{m}{m} \\
\stackrel{i}{i}\end{array}$ & $\begin{array}{l}\text { के } \\
\text { है }\end{array}$ & $\begin{array}{l}\text { త్ర } \\
\stackrel{\sim}{\mathbf{i}}\end{array}$ & $\begin{array}{l}\text { ลิ } \\
\text { ถิ }\end{array}$ & 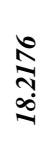 & $\underset{\stackrel{\infty}{\circ}}{\stackrel{\infty}{\circ}}$ \\
\hline 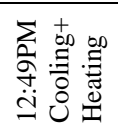 & $\widehat{i}$ & ธี & 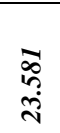 & $\begin{array}{l}\infty \\
\infty \\
\stackrel{2}{2}\end{array}$ & $\underset{\bar{i}}{\bar{i}}$ & 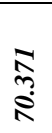 & $\begin{array}{l}\text { 过 } \\
\text { के } \\
\stackrel{0}{-1}\end{array}$ & હે \\
\hline $\begin{array}{l}\sum_{i} \\
\text { in } \\
\text { in }\end{array}$ & 离 & స్ల & $\begin{array}{l}\infty \\
\stackrel{\infty}{n} \\
\sim\end{array}$ & 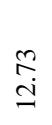 & $\begin{array}{l}\underset{\infty}{0} \\
\stackrel{\Xi}{=}\end{array}$ & $\begin{array}{l}\text { 今े } \\
\text { هి }\end{array}$ & $\begin{array}{l}\frac{2}{6} \\
\stackrel{0}{*}\end{array}$ & 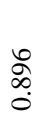 \\
\hline 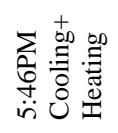 & 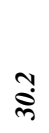 & $\stackrel{\circ}{\circ}$ & $\underset{\grave{d}}{\bar{\sim}}$ & $\overrightarrow{0}$ & 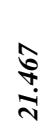 & 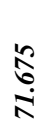 & 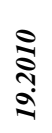 & $\infty$ \\
\hline
\end{tabular}

The required sensible heat from air to evaporate the water, which affects the temperature of the air,is converted into the latent heat and the process is known as adiabatic process as it occurs at constant enthalpy value is shown in the figure 5.1.The cooled and dehumidified air process is also shown on psychromatic chart in figure 5.1.

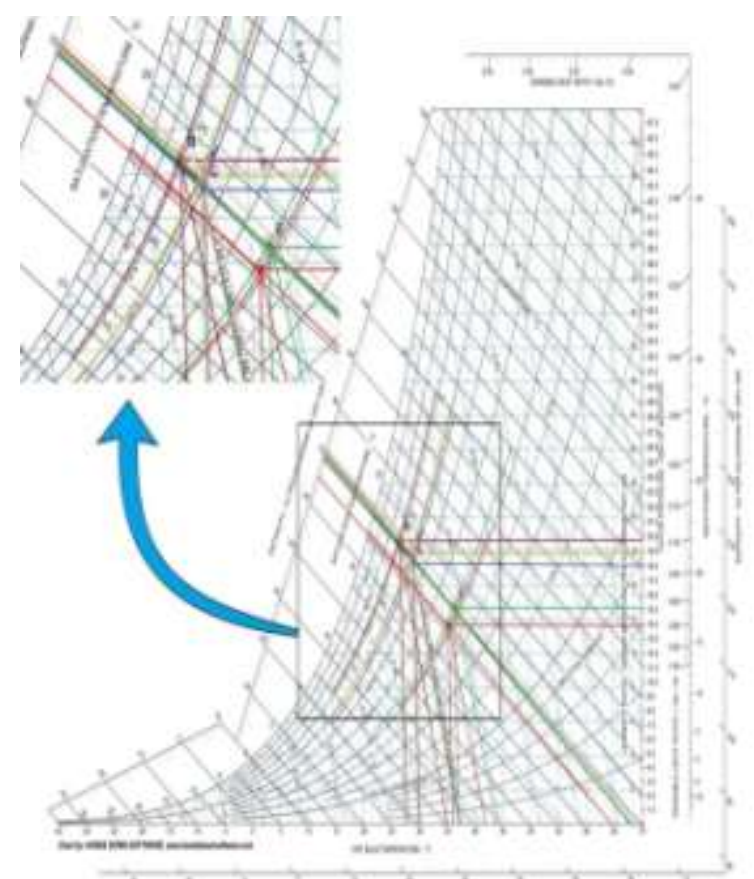

Figure 5.1: Cooled and Dehumidified Air Process

\section{CONCLUSION}

The very commonly used desert cooler in average and below average families do enjoy and feel comfortable in April, May and June especially in Delhi and Rajasthan where day temperature is high and humidity is low as comfort is much related to appropriate level of humidity in atmosphere. Here the desert cooler is modified to control the humidity of the room by increasing the temperature of the air by a heating-coil placed across the cooling pad.This could be obtained by decreasing humidity level and slightly increasing the level of temperature of room. The experiment reveals that by installing desert cooler inside the room, temperature level decrease up to $7^{\circ} \mathrm{C}$ and humidity of the room increase $20 \%$ but here we have decreased the humidity and slightly increased the temperature to obtain the approximately required comfort zone which is suitable for the human being.

\section{ACKNOWLEDGEMENT}

The author is highly thankful to mechanical engineering department for extending help and support in fabricating the experimental set up. The contribution of B Tech final year student viz.Ram Chaudhary,Surya 


\section{REFERENCES}

[1] Faleh Al-Sulaiman, (2002).Evaluation of the performance of local fibers in evaporative cooling. Energy Conversion and Management, Volume 43, Issue 16: pp. 2267-2273.

[2] Poonia M.P., Bhardwaj A., UpenderPandel, JethooA.S., "Design and Development of Energy Efficient Multi-Utility Desert Cooler," Universal Journal of Environmental Research and Technology, Vol 1 39-44, 2011.

[3] Vivek W. Khond, "Experimental investigation of desert cooler performance using four different cooling pad materials," American journal of scientific and industrial research, 2011, 2(3): 418-421

[4] Kothare, C. B. and Borkar, N. B., 2011, "Modified desert cooler (MDC)".International Journal of Engineering and Technology. 3 (2), 166-172

[5] Khond, V. W., 2011, "Experimental investigation of desertcooler performance using four different cooling padmaterials."American Journal of Scientific and Industrial Research.418-421.

[6] RakeshRoshan, UpendraParashar,"Comparative Analysis of Efficiency of Evaporative Air Cooler with Two Different Cooling Pad Material"SSRG International Journal of Thermal Engineering (SSRG IJTE) volume 1 Issue6 November to December 2015

[7] M. Shariaty-Niassar N. Gilani,"An InvestigationofIndirectEvaporativeCoolers,IEC

With Respect to Thermal Comfort Criteria"Iranian Journal of Chemical EngineeringVol. 6, No. 2 (Spring), 2009, IAChE

[8] Vivek W. Khond, "Experimental investigation of desert cooler performance using four different cooling pad materials"American Journal of Scientific and Industrial Research@ 2011, Science Hub, http://www.scihub.org/AJSIRISSN:2153-649X doi:10.5251/ajsir.2011.2.3.418.421

[9] AmarSayed, H., Hiroshi, Y., Abdelsamei Eid, M. and Magdy, M. R. 2012,"Indoor natural ventilation using evaporating cooling strategies in the Egyptian housing: A review and new approach". International Journal of Engineering and Technology. 4(3), 229-233. 